\title{
Research
}

\section{Validity and reliability properties of canine short-term heart rate variability measures-a pilot study}

\author{
Ann Essner ${ }^{\mathrm{a}, \mathrm{b}, *}$, Rita Sjöström ${ }^{\mathrm{c}, \mathrm{d}}$, Pia Gustås ${ }^{\mathrm{e}}$, Laurie Edge-Hughes ${ }^{\mathrm{f}}$, Lena Zetterberg ${ }^{\mathrm{a}}$, \\ Karin Hellström ${ }^{\mathrm{a}}$ \\ a Department of Neuroscience, Section of Physiotherapy, Uppsala University, Uppsala, Sweden \\ ${ }^{\mathrm{b}}$ Evidensia Djurkliniken Gefle, Gävle, Sweden \\ ${ }^{\mathrm{c}}$ Unit of Research, Education and Development, Region Jämtland Härjedalen, Östersund, Sweden \\ ${ }^{\mathrm{d}}$ Department of Health Sciences, Mid Sweden University, Östersund, Sweden \\ e Department of Clinical Sciences, Swedish University of Agricultural Sciences, Uppsala, Sweden \\ ${ }^{\mathrm{f}}$ Canine Fitness Centre, Calgary, Alberta, Canada
}

\section{A R T I C L E I N F O}

Article history:

Received 18 February 2015

Received in revised form

30 April 2015

Accepted 21 May 2015

Available online 17 July 2015

\section{Keywords:}

behavior

dogs

heart rate variability

reliability

validity

\begin{abstract}
A B S T R A C T
The objective of the pilot study was to compare validity and reliability properties of Polar RS800CX (Polar Electro Oy, Kempele, Finland) against simultaneously recorded electrocardiogram (ECG) measuring timeand frequency-based short-term heart rate variability (HRV) parameters, in dogs during stationary standing position. Five-minute recordings with less than $5 \%$ error rates from inter-beat interval (IBI) series obtained by Polar RS800CX and ECG, in 8 adult dogs, were used for HRV analysis. Polar data were statistically compared to the ECG data to assess for systematic differences in time- and frequency-based HRV parameters. Relative and absolute reliabilities were estimated by intraclass correlation coefficient, Spearman $\rho$, Bland and Altman analysis, standard error of measurement, and standard error of measurements in percentage. Paired $t$ test was used to determine the statistical significance of differences between the measurement methods. Results: There were high correlation coefficients between HRV parameters obtained from Polar RS800CX and ECG. Intraclass correlation coefficients were 0.98-1.00, and Spearman $\rho$ was 0.93-0.98. There were differences between the methods in 2 HRV parameters, the standard deviation of normal-to-normal IBIs (SDNN) $(P=0.035)$ and the square root of the mean squared differences of successive normal-to normal IBIs (RMSSD) $(P=0.034)$. Standard error of measurements was between 2.8$11.6 \%$ in ECG and between $2.6-11.8 \%$ in Polar, indicating rather high measurement error in 3 of the HRV parameters in both measurement methods. Close agreements and high correlation estimates in this pilot study indicated acceptable relative reliability in Polar RS800CX measuring time- and frequency-based HRV parameters in the group of dogs studied. However, the present pilot study revealed differences between Polar RS800CX and ECG in time-based standard deviation of normal-to-normal and square root of the mean squared differences of successive normal-to normal parameters, and that small amounts of erroneous IBI segments from Polar negatively impact on the validity and reliability properties of Polar RS800CX.
\end{abstract}

(c) 2015 Elsevier Inc. All rights reserved.

\section{Introduction}

Heart rate variability (HRV) is a biophysiological measure of sympatho-vagal activity in the autonomic nervous system. Within the field of canine behavioral science, the relationship between

\footnotetext{
* Address for reprint requests and correspondence: Ann Essner, Evidensia Djurkliniken Gefle, Norra gatan 1, SE-803 21 Gävle, Sweden, Tel: +46 70 6927562; Fax: +4626106318.

E-mail address: ann.essner@gmail.com (A. Essner).
}

short-term HRV parameters and the level of stress (HydbringSandberg et al., 2004; Bergamasco et al., 2010), responses to human-dog contact (Gácsi et al., 2013; Romero et al., 2013; Tateishi et al., 2014), and physical and mental activities (Kortekaas et al., 2013) have been studied in dogs of various breeds and of differing ages. In humans, reduced parasympathetic cardiac control in HRV analysis has been linked to disorders such as chronic pain in adults (Hallman and Lyskov, 2012; Kang et al., 2012) and children (Evans et al., 2013) and acute pain in newborns (Weissman et al., 2012) and adults (Koenig et al., 2014). HRV as an indicator for the 
bidirectional relationship between autonomic activation and different pain conditions has also been studied in horses (Rietmann et al., 2004), calves (Stewart et al., 2010), and sheep (Stubsjøen et al., 2009). Research on canine autonomic function in relationship to different pathologic conditions (Pirintr et al., 2012; Rasmussen et al., 2012) and assessments on canine cardiac modulation during physical intervention is a matter of great interest because of the potential value as an outcome measure (Wang et al., 2013). As changes in cardiac activity are influenced by psychological and emotional states in dogs (Kuhne et al., 2014a) there are potential clinical applications for short-term HRV parameters as outcome measures for the relief of pain and stress. This concept has been used to study various interventions and exercise regimens for possible effects on the autonomic nervous system in humans (Haker et al., 2000; Anderson et al., 2012; Takamoto et al., 2009; Figueroa et al., 2008; Farinatti et al., 2011; Janse van Rensburg et al., 2012) and in dogs, but to a lesser extent (Wang et al., 2013).

To the authors' knowledge, there are no studies on short-term HRV in dogs with chronic pain. Our ability to conduct clinical studies on different components of pain experiences and levels of stress in dogs, and the study of outcomes resultant from pain management in dogs could increase with the use of non-invasive biomarkers such as short-term HRV (Vainio, 2012).

By studying the variability between cardiac inter-beat intervals (IBIs) in advanced software tools, various HRV parameters may be used to indicate modulations and activity in the autonomic nervous system. To standardize studies on short-term HRV analysis, IBI series of 5 minutes have been recommended (Task Force of the European Society of Cardiology and the North American Society of Pacing, 1996; von Borell et al., 2007; Ille et al., 2014).

HRV may be analyzed in statistical time-based parameters (i.e., variance) and in frequency-based parameters obtained from mathematical algorithms in a power spectral density analysis (Von Borell et al., 2007). There are short-term HRV parameters specifically of interest for the evaluation of interventions targeting the parasympathetic nervous system, as some interventions may potentially reflect the activity in the autonomic nervous system (Koenig et al., 2014). The guidelines on HRV (Task Force of the European Society of Cardiology and the North American Society of Pacing, 1996) specifically recommend the standard deviation of normal-to-normal IBIs (SDNN) and the square root of the mean squared differences of successive normal-to normal IBIs (RMSSD) from the time-based parameters, and low frequency (LF) power, high frequency (HF) power, low frequency power in normalized units (LF n.u.), high frequency power in normalized units (HF n.u.), and the ratio of low frequency power/high frequency power (LF/HF) from the frequency-based parameters in a short-term HRV analysis. To provide information on the contribution of the neural control of heart rate, as in evaluating interventions targeting the parasympathetic nervous system, the RMSSD, HF and HF n.u. are more clinically relevant. The SDNN is an overall measure of HRV and the LF-to-HF ratio has been proposed to provide information on the sympathetic influences of the neural control of heart rate (Thayer, 2009).

Different Polar heart rate monitors have been used to measure heart rate and HRV in canine behavioral research (Kuhne et al., 2014b; Schöberl et al., 2013; Kortekaas et al., 2013; Ogata et al., 2006; Hydbring-Sandberg et al., 2004). The equipment is commercially available, not as expensive to purchase as electrocardiogram (ECG) and easy to apply. Polar heart rate monitors seem to be well accepted by dogs, which is of value for the purpose of recording IBI series for subsequent canine HRV analysis. Series of IBIs for HRV analysis have also been recorded by ECG, and ECG is regarded as the gold standard method for IBI recording (Task Force of the European Society of Cardiology and the North American
Society of Pacing, 1996). Despite the use of Polar RS800CX heart rate monitors to measure canine short-term HRV parameters, there are to the authors' knowledge only a few studies published assessing the validity of Polar RS800CX. Jonckheer-Sheehy et al. (2012) addressed measurement errors and the agreement in IBI measures by Polar RS800CX in their study on 10 stationary Beagle dogs. Another study showed criterion validity and relative reliability were excellent in Polar RS800CX on dogs during standing position and at trot on a treadmill, measuring heart beats per minute (Essner et al., 2013). Criterion validity in Polar RS800CX recording series of IBIs has also been recently reported (Essner et al. 2015). Essner et al. (2015) suggest that IBI series from Polar RS800CX are valid, compared to simultaneously recorded IBIs from ECG, in a group of 8 healthy dogs of different breeds. However, sequences of measurement errors in Polar IBI data have shown to negatively affect the validity of the raw IBI data to be used for subsequent HRV analysis in a group of dogs (Essner et al., 2015).

There appears to be an absence of studies on the reliability properties of time- and frequency-based parameters in short-term HRV analysis from Polar in dogs. Before considering any further clinical application of short-term HRV analysis, using Polar RS800CX in dogs, the relative and absolute reliabilities of the Polar heart rate monitor system, compared to ECG, have to be investigated and reported(Lexell and Downham, 2005; Kottner et al., 2011).

The objective of this pilot study was to compare validity and reliability properties of Polar RS800CX against simultaneously recorded ECG measuring time- and frequency-based short-term HRV parameters, in dogs during stationary standing position.

\section{Methods and materials}

\section{Study design}

This study was an observational study with a methodologically standardized approach (Carter et al., 2011). One group of dogs ( $\mathrm{n}=$ 8 ) with valid and reliable IBI series as reported in a previous study (Essner et al., 2015) was studied with the objective to compare simultaneously recorded IBI series from 2 measurement devices.

\section{Subjects}

Data from 8 ( 3 female and 5 male) dogs of various breeds, with a mean \pm standard deviation (SD) age of $3.5 \pm 1.3$ years, mean \pm SD weight of $32.6 \pm 6.0 \mathrm{~kg}$, and normal body condition were included in the study. None of the dogs had a history or current evidence of cardiovascular or systemic diseases, as assessed by a veterinarian. None of the dogs seemed to react with aggression or fear during the study. As the dogs were privately owned, the owners were informed about the procedures and objectives of the study and informed owner consent was obtained.

\section{Instrumentation and data acquisition}

Polar RS800CX heart rate monitor (Polar Electro Oy, Kempele, Finland) and Cardiostore digital ECG (Vetronic Services Ltd, Abbotskerswell New Abbott, UK) were applied to the dogs as previously described by Essner et al. (2013). One person was responsible for all measurements. The dogs came from their routine activities and were fed not less than 2 hours before the test. The experiment was conducted in a calm environment at a veterinary clinic and at a room temperature of $18^{\circ} \mathrm{C}-22^{\circ} \mathrm{C}$. Recordings were simultaneously started as the dogs were stationary in standing position on an examination table. Polar data were transmitted at the end of each recording to a laptop computer via a bidirectional infrared interface using the Polar software Polar ProTrainer 5. 


\section{Data processing and HRV analysis}

Five-minute recordings from both devices were extracted and visually inspected for artifacts. No nonsinus beats were present in the ECG recordings. The original Polar IBI series with an error count of $5 \%$ or less were used unaltered in the subsequent time- and frequency-based HRV analysis (Task Force of the European Society of Cardiology and the North American Society of Pacing, 1996; von Borell et al., 2007). Polar and Cardiostore software were used to export IBIs as text files to the Windows-based software Kubios HRV 2.0 (Department of Physics, University of Kuopio, Kuopio, Finland) (Niskanen et al., 2004; Tarvainen et al., 2014). Kubios HRV 2.0 was used because it allows for the same HRV analysis technique of both ECG and Polar IBI data in all HRV parameters chosen. Data for analysis were derived from the first 300-second IBI segment in the ECG and the Polar IBI series respectively from each subject. Kubios HRV generated a power spectral density analysis using fast Fourier transform (Parker et al., 2010; Jonckheer-Sheehy et al., 2012; Tateishi et al., 2014; Kuhne et al., 2014b), a Welsh periodogram with 256 seconds window and 50\% overlap. Frequency-based parameters selected were low frequency (LF, 0.04-0.15 cycles/beat), high frequency (HF, 0.15-0.60 cycles/beat), low frequency power in normalized units (LF n.u.), high frequency power in normalized units (HF n.u.), and ratio low frequency power/high frequency power $(\mathrm{LF} / \mathrm{HF})$. Selected time-based parameters were SDNN and RMSSD.

\section{Statistical analysis}

Estimated HRV parameters within the group of dogs studied were analyzed with SPSS (Version 20, IBM Statistical Package for Social Science Statistics for Windows, Armonk, NY: IBM Corp). Pairwise HRV data based on IBIs from ECG and Polar were used to examine if any significant $(P<0.05)$ systematic bias existed between the parameters analyzed. Paired $t$ test was used in all HRV parameters to determine the statistical significance of differences between the measurement methods. No corrections for multiple tests were performed.

The correlations between the methods and the relative reliabilities of Polar RS800CX were estimated by using Spearman $\rho$, and an intraclass correlation coefficient (ICC) (Shrout and Fleiss, 1979), with a 95\% confidence interval (CI). Specifically, the ICC chosen was of a single measure and in absolute agreement with 2way random effects $\left(\mathrm{ICC}_{2.1}\right)$. A correlation coefficient based on ranks (i.e., Spearman $\rho$ ) was chosen in addition to ICC as it is less affected by outliers in the data (Estelberger and Reibnegger, 1995). Hopkins (2000) suggested a correlation coefficient of $>0.81$ is desirable. Shrout and Fleiss (1979) claimed that ICC $>0.75$ indicates excellent reliability.

Absolute reliabilities were investigated by calculating the standard error of measurements (SEM) and SEM\% (Hopkins, 2000; Atkinson and Nevill, 1998) in Polar and ECG measurements, respectively. Estimates of SEM were represented in the same unit as the original measurement for each HRV parameter selected and were calculated according to (Atkinson and Nevill, 1998):

$$
\mathrm{SEM}=\mathrm{SD} \sqrt{1-\mathrm{ICC}_{2.1}}
$$

$\mathrm{SEM} \%$ was defined as SEM $\%=\mathrm{SEM} /$ mean $\times 100$, whereby mean was the average of measures from Polar and ECG, respectively.

Finally, Bland and Altman plots with 95\% limits of agreement (LoA) and 95\% CI of mean differences were constructed to examine the level of agreement between ECG and Polar HRV parameters. The presence of any systematic overestimation and underestimation of time- and frequency-based parameters was assessed, and the upper and lower LoA were calculated by the $\mathrm{SD} \pm 1.96$ of the mean difference between methods (Bland and Altman, 1999).

\section{Results}

Eight dogs completed the pilot study and provided data from the recording of the IBI series. Summary statistics of time- and frequency-based parameters of HRV analysis from Polar and ECG HRV analysis are presented in Table 1.

\section{Relationship between HRV parameters recorded from Polar and ECG}

The correlations between Polar and ECG varied slightly among HRV variables, although Spearman $\rho$ and $\mathrm{ICC}_{2.1}$ showed overall very strong correlations between HRV parameters in all time- and frequency-based parameters selected. SDNN, RMSDD, LF, HF, LF n.u., HF n.u., and LF/HF correlation coefficients are presented in Table 2. In addition, $95 \% \mathrm{CI}$ for $\mathrm{ICC}_{2.1}$ was narrow and ranging from 0.85 to 1.00 , indicating that the true difference between these measures was small (Table 2).

\section{Differences between HRV parameters recorded from Polar and ECG}

The time-based parameters obtained from ECG and Polar data indicated there was a difference between ECG and Polar in SDNN $(P=$ $0.035)$ and $\operatorname{RMSSD}(P=0.034)$. There were no differences in the frequency-based parameters from the HRV analysis $(P>0.05)$ (Table 1$)$.

Within-group variation and agreement in Polar and ECG measurements

Percentage measurement reliability (SEM\%) varied between $2.8 \%$ and $11.6 \%$ in ECG and between $2.6 \%$ and $11.8 \%$ in Polar, indicating the levels of accuracy varied between low and high among HRV variables. The absolute reliabilities of each HRV parameter in Polar and ECG, estimated by the SEM and SEM\%, are shown in Table 2. There were large within-group variations observed in both time- and frequency-based HRV parameters in both measurement methods.

Mean differences showed that the Polar was both overestimating and underestimating the HRV parameters, compared to ECG. In both time-based HRV parameters, SDNN and RMSSD, the differences between Polar and ECG were within LoA. Although, in the frequency-based parameters the Bland and Altman plots indicated that the majority, but $1<95 \%$ of the differences were within LoA in LF, LF n.u. and HF n.u. Table 3 summarizes mean differences, $\mathrm{SD}$ of the differences, LoA, and the CI of the mean differences between measurement methods. The Bland and Altman plots of the

Table 1

Summary statistics of time- and frequency-based parameters of heart rate variability analysis from ECG and Polar HRV data $(\mathrm{n}=8)$

\begin{tabular}{lccl}
\hline HRV parameter & Polar, M (SD) & ECG, M (SD) & $P$-value \\
\hline SDNN (ms) & $70.5(18.4)$ & $72.5(20.1)$ & $0.035^{\mathrm{a}}$ \\
RMSSD (ms) & $54.4(32.9)$ & $58.6(37.1)$ & $0.034^{\mathrm{a}}$ \\
LF $\left(\mathrm{ms}^{2}\right)$ & $1411.1(1045.2)$ & $1443.4(1028.5)$ & 0.298 \\
HF $\left(\mathrm{ms}^{2}\right)$ & $1486.8(1758.8)$ & $1653.3(1918.2)$ & 0.061 \\
LF n.u. & $55.9(18.5)$ & $54.4(18.9)$ & 0.299 \\
HF n.u. & $44.1(18.5)$ & $45.6(18.9)$ & 0.223 \\
LF/HF & $1.8(1.5)$ & $1.7(1.5)$ & 0.223 \\
\hline
\end{tabular}

HF, power in the high frequency range; HF n.u., high frequency power in normalized units; LF, power in the low frequency range; LF n.u., low frequency power in normalized units; LF/HF, ratio low frequency power/high frequency power; $M$, mean; RMSSD, root mean square of successive differences; SD, standard deviation; SDNN, mean of standard deviation of normal-to-normal intervals.

Significance of the difference between Polar and ECG, $P<0.05$.

a Represent significant difference in comparison. 
Table 2

Reliability estimates of Polar, compared to ECG, measuring time- and frequencybased heart rate variability parameters in dogs during stationary standing position

\begin{tabular}{lllrrrr}
\hline $\begin{array}{l}\text { HRV } \\
\text { parameter }\end{array}$ & $\begin{array}{l}\text { Spearman } \\
\rho\end{array}$ & $\mathrm{ICC}_{2.1}(\mathrm{CI})$ & $\begin{array}{l}\text { SEM } \\
\text { ECG }\end{array}$ & $\begin{array}{l}\text { SEM\% } \\
\text { ECG }\end{array}$ & $\begin{array}{l}\text { SEM } \\
\text { Polar }\end{array}$ & $\begin{array}{l}\text { SEM\% } \\
\text { Polar }\end{array}$ \\
\hline SDNN (ms) & 0.95 & $0.99(0.90-1.00)$ & 2.0 & 2.8 & 1.8 & 2.6 \\
RMSSD (ms) & 0.98 & $0.99(0.85-1.00)$ & 4.5 & 7.7 & 4.0 & 7.4 \\
LF $\left(\mathrm{ms}^{2}\right)$ & 0.93 & $1.00(0.99-1.00)$ & 56.3 & 3.9 & 57.2 & 4.1 \\
HF $\left(\mathrm{ms}^{2}\right)$ & 0.95 & $0.99(0.93-1.00)$ & 191.8 & 11.6 & 175.9 & 11.8 \\
LF n.u. & 0.93 & $0.98(0.93-1.00)$ & 2.4 & 4.4 & 2.3 & 4.1 \\
HF n.u. & 0.93 & $0.98(0.93-1.00)$ & 2.4 & 5.3 & 2.3 & 5.2 \\
LF/HF & 0.93 & $0.99(0.96-1.00)$ & 0.2 & 9.0 & 0.2 & 8.6 \\
\hline
\end{tabular}

$\mathrm{CI}$, confidence interval (lower and upper); HF, power in the high frequency range; HF n.u., high frequency power in normalized units; ICC, intraclass correlation coefficient; LF, power in the low frequency range; LF n.u., low frequency power in normalized units; LF/HF, ratio low frequency power/high frequency power; RMSSD, root mean square of successive differences; SDNN, mean of standard deviation of normal-to-normal intervals; SEM, standard error of measurement; SEM\%, standard error of the measurement expressed as a percentage.

differences between the ECG and Polar against their mean HRV values are shown in Figure 1 A,B and Figure 2 A-E.

\section{Discussion}

In the present pilot study, we examined the validity and reliability properties of Polar RS800CX by comparing the heart rate monitor against simultaneous ECG in a group of dogs. Our pilot study is the first to provide estimates on the criterion validity and instrument reliability properties of Polar RS800CX measuring short-term HRV parameters in a group of dogs from various breeds. Absolute and relative reliabilities of Polar RS800CX were estimated on a number of HRV parameters previously used within canine behavioral research and within human pain and stress science. Overall high ICC (0.98-1.00) and narrow CIs showed that the HRV parameters were strongly associated between measurement methods. In addition, we estimated the correlation coefficients by Spearman $\rho$, as there were outliers present in the SDNN, LF, HF, and $\mathrm{LF} / \mathrm{HF}$ parameters. The correlation coefficients by Spearman $\rho$ were lower (0.93-0.98) yet very high.

In this study, the Bland and Altman analysis indicated that Polar underestimated the time-based HRV parameters. Although statistically significant for the SDNN ( $-1.9 \mathrm{~ms})$ and RMSSD ( $-4.3 \mathrm{~ms})$, the
Table 3

Mean (M) differences between Polar and ECG (Polar minus ECG) in heart rate variability parameters selected \pm standard deviation (SD) of the differences, 95\% limits of agreement, and $95 \%$ confidence intervals (CI)

\begin{tabular}{lccr}
\hline HRV parameter & M (SD) differences & 95\% LoA & \multicolumn{1}{c}{$95 \% \mathrm{CI}$} \\
\hline SDNN (ms) & $-1.9(2.1)$ & -6.0 to 2.2 & -3.6 to -0.2 \\
RMSSD (ms) & $-4.3(4.6)$ & -13.3 to 4.8 & -8.2 to -0.4 \\
LF $\left(\mathrm{ms}^{2}\right)$ & $-32.3(81.2)$ & -191.4 to 126.9 & -100.2 to 35.7 \\
HF $\left(\mathrm{ms}^{2}\right)$ & $-166.5(211.6)$ & -581.3 to 248.3 & -343.4 to 10.4 \\
LF n.u. & $1.5(3.2)$ & -4.8 to 7.9 & -1.2 to 4.2 \\
HF n.u. & $-1.5(3.3)$ & -7.9 to 4.8 & -4.2 to 1.2 \\
LF/HF & $0.1(0.2)$ & -0.3 to 0.5 & -0.1 to 0.3 \\
\hline
\end{tabular}

$\mathrm{CI}$, confidence interval (lower and upper); $\mathrm{HF}$, power in the high frequency range; $\mathrm{HF}$ n.u., high frequency power in normalized units; LF, power in the low frequency range; $\mathrm{LF}$ n.u., low frequency power in normalized units; LF/HF, ratio low frequency power/high frequency power; LoA, limits of agreement (lower and upper); RMSSD, root mean square of successive differences; SDNN, mean of standard deviation of normal-to-normal intervals.

underestimation may not be clinically significant. The SDNN and RMSSD measurements were all within the 95\% upper and lower LoA, and in comparison to the HRV values previously recorded in a study by Gácsi et al. (2013) the mean difference in SDNN was low. In the frequency-based parameters, LF, LF n.u., and HF n.u., there was 1 measurement in each parameter outside LoA. It should be pointed out that the study group was rather small and the LoA may be biased by the sample size. There is a need for a larger sample to learn more about the level of agreement between Polar and ECG measuring HRV, based on a Bland and Altman analysis.

In contradiction to Jonckheer-Sheehy et al. (2012), the estimated differences between Polar and ECG were significant in 2 of the HRV parameters. Paired t test revealed that there were differences between Polar and ECG in SDNN $(P=0.035)$ and RMSSD $(P=0.034)$. Jonckheer-Sheehy et al. (2012) compared time- and frequency-based HRV parameters in 10 Beagle dogs and found that there were no significant differences between Polar and ECG, although they corrected their estimations for multiple tests. We are aware of the increased probability of statistical type 1 error with each additional test performed in the present study. Rather than to compensate for multiple tests by dividing alpha, we chose to not reduce the power of each test by doing so. Except for the analysis of the differences between Polar and ECG made by Jonckheer-Sheehy et al. (2012), there
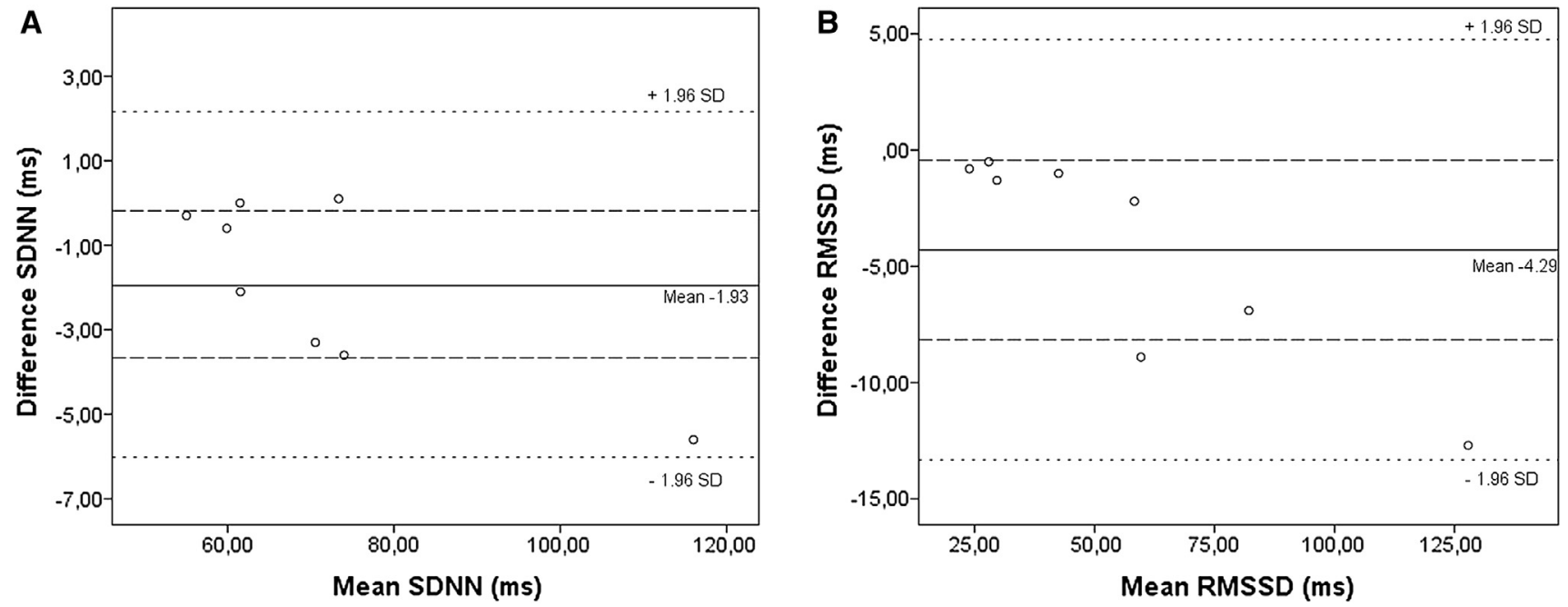

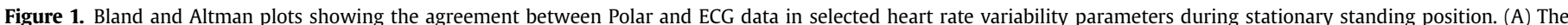

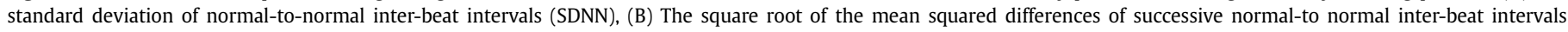

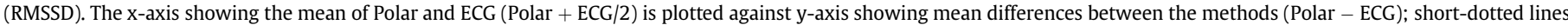

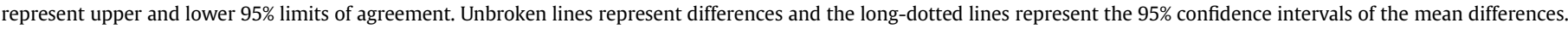



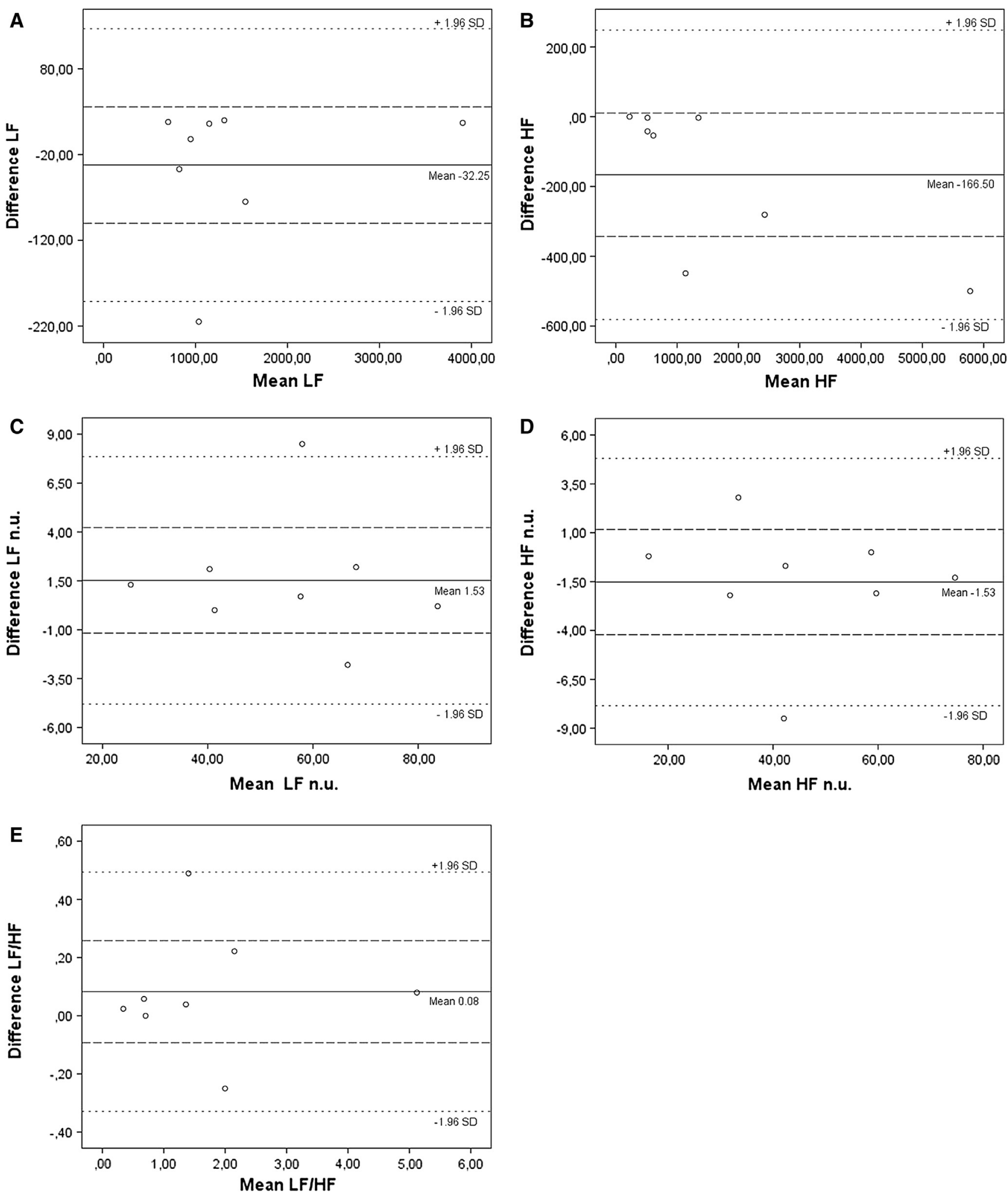

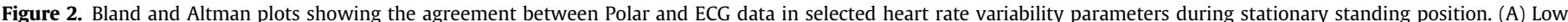

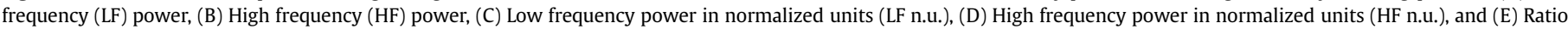

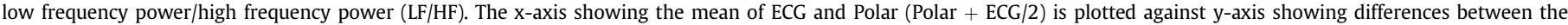

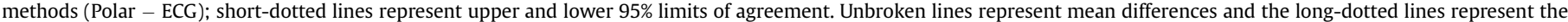
$95 \%$ confidence intervals of the mean differences.

are no other comparable validity and reliability testing performed on Polar RS800CX in dogs before the present study. As our pilot study indicated differences between HRV parameters recorded by Polar
RS800CX and ECG, the next step may be to use an algorithmsupported visual error correction in the Polar IBI series (Schöberl et al., 2014). The IBI series used in the present pilot study 
contained less than $5 \%$ of erroneous data, and yet the small amount of errors had negative impact on the criterion validity and reliability properties of Polar RS800CX HRV parameters. Therefore, our study supports further testing of the algorithm-supported visual error correction recently presented by Schöberl et al. (2014).

In order for the results of a recording device to be applicable for evaluating short-term HRV parameters, the validity of the device has to be greater than the measurement error. Low levels of SEM indicate high levels of accuracy and high levels of SEM indicate low accuracy. Interestingly, the calculations of the absolute reliabilities in the present study demonstrated that SEM and SEM\% values in the data obtained with the Polar RS800CX were close to the measurement errors obtained by ECG. Rather similar and high SEM and SEM\% in Polar and ECG are possibly due to the large within-group variations in both measurement methods. Standard error of measurements in percentage varied between the HRV parameters in Polar (2.6-11.8\%) and in ECG (2.8-11.6\%) showing that SDNN, LF, and LF n.u. are HRV parameters in which the minimal detectable difference may be easier to find than in other parameters.

We assume there is more to be investigated concerning shortterm HRV analysis performed in dogs, as SEM and SEM\% were sometimes high even in the ECG estimates, implying low accuracy in group level data. Comparison of behavioral and physiological data across individuals has been discussed previously by Koolhaas et al. (2010). The interpretation of research results is challenged by the presence of individual variations that needs to be considered when implementing canine HRV studies. Hopkins (2000) showed that even a strictly standardized protocol may be influenced by biological within-subject variations. Age, sex, breed, and circadian rhythm may influence canine HRV parameters (Olsen et al., 1999; Doxey and Boswood, 2004; Nolan et al., 2004). Inherited individual variations, breathing frequency, and physical human-dog interaction are other examples of possible factors influencing canine HRV measures (Koolhaas et al., 2010; Minors and O'Grady, 1997; Kuhne et al., 2014b). As this pilot study was observational, no causalities may be drawn about any confounding variables in our sample. It was outside the objectives of this study to assess the background of confounding influences.

As the present study revealed large within-group variations in both ECG and Polar data, we suggest that canine HRV researchers consider the use of multiple subjects in single-case experimental designs (Kazdin, 2002; Koolhaas et al., 2010). Large variability among HRV parameters in a group of dogs will inevitably lead to larger SEM and SEM\% and reduced possibility to detect true differences in group data. We recommend attempts to control for variables known to influence HRV in future canine HRV research. However the individual variations in biophysiological measures may serve an evolutionary function in nature, and they also commonly exist in veterinary and physiotherapeutic clinical practice. Hence, research within different disciplines (i.e., anthrozoology; biomedicine; behavior science; physiotherapy; veterinary medicine) will probably continue to observe individual differentiations in response to varying interventions (Koolhaas et al., 2010). To use HRV measures in single-case experimental designs, the testretest reliability (i.e., repeatability) of short-term HRV measures has to be further explored in dogs (Sandercock et al., 2005; Brown et al., 1989). There is a need for a study design other than ours for the purpose of assessing the test-retest reliability and confounding variables in canine short-term HRV analysis.

Additionally, we look forward to studies implementing HRV measures in assessing sympatho-vagal activity and canine chronic pain behavior, as there are studies suggesting that conditions perceived as unpleasant by dogs may influence short-term HRV measures (Kuhne et al., 2014b; Schöberl et al., 2013).

\section{Conclusion}

Close agreements and high correlation estimated in the present pilot study indicated acceptable relative reliability in Polar RS800CX measuring time- and frequency-based HRV parameters in the group of dogs studied. The absolute reliability estimated from Polar and ECG showed that it may be difficult to monitor small changes in some of the canine HRV parameters in group level data, because of large within-group variations. Our pilot study revealed differences between Polar RS800CX and ECG, and that small amounts of erroneous IBI segments from Polar may negatively impact on the validity and reliability properties of Polar RS800CX.

\section{Acknowledgments}

We are grateful to the dogs and their owners who participated in this study. Special thanks also to Erik Ahlgren and Marianne Mellgren for excellent support to carry out the study. The study was approved by the Local Ethical Committee in Uppsala (C81/12). The study was supported by a grant from Svelands stiftelse för djurens hälsa och livskvalité, Sweden. All authors contributed to the article. The idea for the article was conceived by Ann Essner. Ann Essner and Rita Sjöström made substantial contributions to conception and design of the article. Ann Essner was responsible for the data acquisition and processed the data. In addition, Ann Essner was the main author and Rita Sjöström, Pia Gustås, Laurie Edge-Hughes, Lena Zetterberg, and Karin Hellström contributed in drafting the article and revising it critically. All authors analyzed the data, discussed the results and the interpretation of the results, commented on the article, and gave final approval of the version to be published.

\section{Conflict of interest}

Authors disclose no conflict of interest.

\section{References}

Anderson, B., Nielsen, A., McKee, D., Jeffres, A., Kligler, B., 2012. Acupuncture and heart rate variability: a systems level approach to understanding mechanism. Explore (NY) 8, 99-106.

Atkinson, G., Nevill, A.M., 1998. Statistical methods for assessing measurement error (reliability) in variables relevant to sports medicine. Sports Med. 26, 217-238.

Bergamasco, L., Osella, M., Savarino, P., Larosa, G., Ozella, L., Manassero, M. Badino, P., Odore, R., Barbero, R., Re, G., 2010. Heart rate variability and saliva cortisol assessment in shelter dog: human-animal interaction effects. Appl. Anim. Behav. Sci. 125, 56-68.

Bland, J.M., Altman, D.G., 1999. Measuring agreement in method comparison studies. Stat. Methods Med. Res. 8, 135-160.

Brown, D.R., Randall, D.C., Knapp, C.F., Lee, K.C., Yingling, J.D., 1989. Stability of the heart rate power spectrum over time in the conscious dog. FASEB J. 3, 16441650.

Carter, R.E., Lubinsky, J., Domholdt, E., 2011. Rehabilitation Research, 4th ed. Elsevier Saunders, St. Louis.

Doxey, S., Boswood, A., 2004. Differences between breeds of dog in a measure of heart rate variability. Vet. Rec. 154, 713-717.

Essner, A., Sjöström, R., Ahlgren, E., Gustås, P., Edge-Hughes, L., Zetterberg, L., Hellström, K., 2015. Comparison of Polar ${ }^{\circledR}$ RS800CX heart rate monitor and electrocardiogram for measuring inter-beat intervals in healthy dogs. Physiol. Behav. 138, 247-253.

Essner, A., Sjöström, R., Ahlgren, E., Lindmark, B., 2013. Validity and reliability of Polar ${ }^{\circledR}$ RS800CX heart rate monitor, measuring heart rate in dogs during standing position and at trot on a treadmill. Physiol. Behav., 114-115, 1-5.

Estelberger, W., Reibnegger, G., 1995. The rank correlation coefficient: an additional aid in the interpretation of laboratory data. Clin. Chim. Acta. 239, 203-207.

Evans, S., Seidman, L.C., Tsao, J.C., Lung, K.C., Zeltzer, L.K., Naliboff, B.D., 2013. Heart rate variability as a biomarker for autonomic nervous system response differences between children with chronic pain and healthy control children. J. Pain Res. 6, 449-457.

Farinatti, P., Brandão, C., Soares, P., Duarte, A., 2011. Acute effects of stretching exercise on the heart rate variability in subjects with low flexibility levels. J. Strength Cond. Res. 25, 1579-1585. 
Figueroa, A., Kingsley, J.D., McMillan, V., Panton, L.B., 2008. Resistance exercise training improves heart rate variability in women with fibromyalgia. Clin. Physiol. Funct. Imaging 28, 49-54.

Gácsi, M., Maros, K., Sernkvist, S., Faragó, T., Miklósi, A., 2013. Human analogue safe haven effect of the owner: behavioural and heart rate response to stressful social stimuli in dogs. PLoS One 8, e58475.

Haker, E., Egekvist, H., Bjerring, P., 2000. Effect of sensory stimulation (acupuncture) on sympathetic and parasympathetic activities in healthy subjects. J. Auton. Nerv. Syst. 79, 52-59.

Hallman, D.M., Lyskov, E., 2012. Autonomic regulation, physical activity and perceived stress in subjects with musculoskeletal pain: 24-hour ambulatory monitoring. Int. J. Psychophysiol. 86, 276-282.

Hopkins, W.G., 2000. Measures of reliability in sports medicine and science. Sports Med. 30, 1-15.

Hydbring-Sandberg, E., von Walter, L.W., Hoglund, K., Svartberg, K., Swenson, L., Forkman, B., 2004. Physiological reactions to fear provocation in dogs. J. Endocrinol. 180, 439-448.

Ille, N., Erber, R., Aurich, C., Aurich, J., 2014. Comparison of heart rate and heart rate variability obtained by heart rate monitors and simultaneously recorded electrocardiogram signals in nonexercising horses. J. Vet. Behav.: Clin. Appl. Res. 9, 341-346.

Janse van Rensburg, D.C., Ker, J.A., Grant, C.C., Fletcher, L., 2012. Effect of exercise on cardiac autonomic function in females with rheumatoid arthritis. Clin. Rheumatol. 31, 1155-1162.

Jonckheer-Sheehy, V., Vinke, C., Ortolani, A., 2012. Validation of a Polar ${ }^{\circledR}$ human heart rate monitor for measuring heart rate and heart rate variability in adult dogs under stationary conditions. J. Vet. Behav.: Clin. Appl. Res. 7, 205-212.

Kang, J.H., Chen, H.S., Chen, S.C., Jaw, F.S., 2012. Disability in patients with chronic neck pain: heart rate variability analysis and cluster analysis. Clin. J. Pain 28, 797-803.

Kazdin, A.E., 2002. Research Design in Clinical Psychology, 4th ed. Pearson, Boston.

Koenig, J., Jarczok, M.N., Ellis, R.J., Hillecke, T.K., Thayer, J.F., 2014. Heart rate variability and experimentally induced pain in healthy adults: a systematic review. Eur. J. Pain 18, 301-314.

Koolhaas, J.M., de Boer, S.F., Coppens, C.M., Buwalda, B., 2010. Neuroendocrinology of coping styles: towards understanding the biology of individual variation. Front. Neuroendocrinol. 31, 307-321.

Kortekaas, K., Range, F., Virányi, Z., Kotrschal, K., 2013. Heart rate and heart rate variability of dogs (Canis lupus familiaris) during physical and mental activities. J. Vet. Behav.: Clin. Appl. Res. 8, e45-e46.

Kottner, J., Audigé, L., Brorson, S., Donner, A., Gajewski, B., Hróbjartsson, A., Roberts, C., Shoukri, M., Streiner, D., 2011. Guidelines for reporting reliability and agreement studies (GRRAS) were proposed. J. Clin. Epidemiol. 64, 96-106.

Kuhne, F., Hößler, J.C., Struwe, R., 2014a. Emotions in dogs being petted by a familiar or unfamiliar person: validating behavioural indicators of emotional states using heart rate variability. Appl. Anim. Behav. Sci. 161, 113-120. http:// dx.doi.org/10.1016/j.applanim.2014.09.020.

Kuhne, F., Hößler, J.C., Struwe, R., 2014b. Behavioral and cardiac responses by dogs to physical human-dog contact. J. Vet. Behav.: Clin. Appl. Res. 9, 93-97.

Lexell, J.E., Downham, D.Y., 2005. How to assess the reliability of measurements in rehabilitation. Am. J. Phys. Med. Rehabil. 84, 719-723.

Minors, S.L., O'Grady, M.R., 1997. Heart rate variability in the dog: is it too variable? Can. J. Vet. Res. 61, 134-144.

Niskanen, J.P., Tarvainen, M.P., Ranta-Aho, P.O., Karjalainen, P.A., 2004. Software for advanced HRV analysis. Comput. Methods Programs Biomed. 76, 73-81.

Nolan, E.R., Girand, M., Bailie, M., Yeragani, V.K., 2004. Circadian changes in the QT variability index in the beagle dog. Clin. Exp. Pharmacol. Physiol. 31, 783-785.

Ogata, N., Kikusui, T., Takeuchi, Y., Mori, Y., 2006. Objective measurement of fearassociated learning in dogs. J. Appl. Phys. 1, 55-61.

Olsen, L.H., Mow, T., Koch, J., Pedersen, H.D., 1999. Heart rate variability in young, clinically healthy Dachshunds: influence of sex, mitral valve prolapse status, sampling period and time of day. J. Vet. Cardiol. 1, 7-16.
Parker, M., Goodwin, D., Eager, R.A., Redhead, E.S., Marlin, D.J., 2010. Comparison of Polar ${ }^{\circledR}$ heart rate interval data with simultaneously recorded ECG signals in horses. Comp. Exerc. Physiol. 6, 137-142.

Pirintr, P., Chansaisakorn, W., Trisiriroj, M., Kalandakanond-Thongsong, S. Buranakarl, C., 2012. Heart rate variability and plasma norepinephrine concentration in diabetic dogs at rest. Vet. Res. Commun. 36, 207-214.

Rasmussen, C.E., Falk, T., Zois, N.E., Moesgaard, S.G., Häggström, J., Pedersen, H.D., Ablad, B., Nilsen, H.Y., Olsen, L.H., 2012. Heart rate, heart rate variability, and arrhythmias in dogs with myxomatous mitral valve disease. J. Vet. Intern. Med. 26, 76-84.

Rietmann, T.R., Stauffacher, M., Bernasconi, P., Auer, J.A., Weishaupt, M.A., 2004. The association between heart rate, heart rate variability, endocrine and behavioura pain measures in horses suffering from laminitis. J. Vet. Med. Physiol. Pathol. Clin. Med. 51, 218-225.

Romero, T., Konno, A., Hasegawa, T., 2013. Familiarity bias and physiological responses in contagious yawning by dogs support link to empathy. PLoS One 8 e71365.

Sandercock, G.R., Bromley, P.D., Brodie, D.A., 2005. The reliability of short-term measurements of heart rate variability. Int. J. Cardiol. 103, 238-247.

Schöberl, I., Kortekaas, K., Schöberl, F.F., Kotrschal, K., 2014. Algorithm-supported visual error correction (AVEC) of heart rate measurements in dogs, Canis lupus familiaris. Behav. Res. Methods. [Epub ahead of print]. http://dx.doi.org/ 10.3758/s13428-014-0546-Z.

Schöberl, I., Wedl, M., Kotrschal, K., 2013. Heart rate and heart rate variability in owners and their dogs. J. Vet. Behav.: Clin. Appl. Res. 8, e34.

Shrout, P.E., Fleiss, J.L., 1979. Intraclass correlations: Uses in assessing rater reliability. Psychol. Bull. 86, 420-428.

Stewart, M., Verkerk, G.A., Stafford, K.J., Schaefer, A.L., Webster, J.R., 2010. Noninvasive assessment of autonomic activity for evaluation of pain in calves, using surgical castration as a model. J. Dairy Sci. 93, 3602-3609.

Stubsjøen, S.M., Flø, A.S., Moe, R.O., Janczak, A.M., Skjerve, E., Valle, P.S., Zanella, A.J., 2009. Exploring non-invasive methods to assess pain in sheep. Physiol. Behav. 98, 640-648.

Takamoto, K., Sakai, S., Hori, E., Urakawa, S., Umeno, K., Ono, T., Nishijo, H., 2009. Compression on trigger points in the leg muscle increases parasympathetic nervous activity based on heart rate variability. J. Physiol. Sci. 59, $191-197$.

Tarvainen, M.P., Niskanen, J.P., Lipponen, J.A., Ranta-aho, P.O., Karjalainen, P.A., 2014 Kubios HRV - Heart rate variability analysis software. Comput. Methods Programs Biomed. 113, 210-220.

Task Force of the European Society of Cardiology and the North American Society of Pacing, 1996. Heart rate variability: standards of measurement, physiological interpretation, and clinical use. Eur. Heart J. 17, 354-381.

Tateishi, K., Ohtani, N., Ohta, M., 2014. Physiological effects of interactions between female dog owners with neuroticism and their dogs. J. Vet. Behav.: Clin. Appl. Res. 9, 304-310.

Thayer, J.F., 2009. Heart rate variability: a neurovisceral integration model. In: Squire, L.R. (Ed.), Encyclopedia of Neuroscience. Academic Press, Oxford, pp. 1041-1047.

Vainio, O., 2012. Translational animal models using veterinary patients-an example of canine osteoarthritis (OA). Scand. J. Pain 3, 84-89.

Von Borell, E., Langbein, J., Després, G., Hansen, S., Leterrier, C., Marchant-Forde, J., Marchant-Forde, R., Minero, M., Mohr, E., Prunier, A., Valance, D., Veissier, I., 2007. Heart rate variability as a measure of autonomic regulation of cardiac activity for assessing stress and welfare in farm animals - a review. Review. Physiol. Behav. 92, 293-316.

Wang, H., Litscher, G., Shi, X.J., Jiang, Y.B., Wang, L., 2013. Effects of acupuncture on heart rate variability in beagles; preliminary results. Evid. Based Complement Altern. Med. 2013. http://dx.doi.org/10.1155/2013/419212.

Weissman, A., Zimmer, E.Z., Aranovitch, M., Blazer, S., 2012. Heart rate dynamics during acute pain in newborns. ü Arch. 464, 593-599. 